\title{
General perturbative approach to the diffusion equation in the presence of absorbing defects: Frequency-domain and time-domain results
}

\author{
Angelo Sassaroli ${ }^{\mathrm{a}}$, Fabrizio Martelli ${ }^{\mathrm{b}}$, and Sergio Fantini ${ }^{\mathrm{a}}$ \\ ${ }^{a}$ Department of Biomedical Engineering, Tufts University, \\ 4 Colby Street, Medford, MA 02155 \\ ${ }^{\mathrm{b}}$ Dipartimento di Fisica dell’Università degli Studi di Firenze, \\ Via G. Sansone 1,50019 Sesto Fiorentino, Firenze, Italy
}

\begin{abstract}
In this work, based on our previously proposed perturbation theory for the diffusion equation, we present new theoretical results in time and frequency domains. More specifically, we have developed a fourth order perturbation theory of the diffusion equation for absorbing defects. The method of Padé Approximants is used to extend the validity of the proposed theory to a wider range of absorbing contrasts between defects and background medium. The results of the theory are validated by comparisons with Monte Carlo simulations. In the frequency domain, the discrepancy between theoretical and Monte Carlo results for amplitude ( $A C$ ) data are less than $10 \%$ up to an absorption contrast of $\Delta \mu_{a} \leq 0.2 \mathrm{~mm}^{-1}$, whereas the discrepancy of phase data is less than $1^{\circ}$ up to $\Delta \mu_{a} \leq 0.1 \mathrm{~mm}^{-1}$. In the time domain, the average discrepancy is around 2-3\% up to $\Delta \mu_{a} \leq 0.06 \mathrm{~mm}^{-1}$. The proposed method is an effective and fast forwardproblem solver that has the potential to find general applicability in a number of situations.
\end{abstract}

\section{INTRODUCTION}

In Near Infra-Red Spectroscopy (NIRS) and Diffuse Optical Tomography (DOT), one of the main challenges is represented by modeling light propagation in biological tissues. Currently, a wide variety of methods have been used: analytical methods ${ }^{1-2}$, Finite Element Methods (FEM) for solving the diffusion equation $^{3-5}(\mathrm{DE})$, a combination of the discrete ordinate method and the finite volume method for solving the more general Radiative Transfer Equation ${ }^{6}$ (RTE) etc.. Numerical methods can be applied to very general reconstruction problems, regardless of the geometry of the medium and distribution of the optical properties; however they are characterized by long computation times and sensitivity to artifacts. Analytical methods are much simpler but they are limited to the cases of homogeneous or layered media bounded by regular surfaces. They have been successfully used on phantom studies for optical properties measurements and also as approximate methods for the estimation of the bulk optical properties of tissues in some in vivo experiments. Among analytical methods, one can also include those based on perturbation theory of the $\mathrm{DE}$, which have been proposed for solving problems of light propagation in media characterized by focal changes in the optical properties. Here we remind pioneer studies by Ostermeyer et al. ${ }^{7}$ and $\mathrm{Boas}^{8}$, in the frequency domain, and more recent works in the time domain ${ }^{9-10}$. In the aforementioned publications, the authors have also shown the possibility to apply an iterative method for the calculation and update of the fluence rate in order to obtain an $n^{\text {th }}$ order perturbation theory. Higher order perturbation theory overcomes the limitation of first order theory (Born approximation) rendering it suitable for solving problems where the defects embedded in the medium are not "small." More precisely, $n^{\text {th }}$ order perturbation theory can be applied when the change in the detected intensity with respect to the initial medium (not including the defects) lies outside the region of linearity. In all published studies on perturbation theory, the authors have developed more or less sophisticated mathematical methods to find the solution of the DE for both absorbing and scattering defects in particular geometries. In our works, ${ }^{11-15}$ we focused mainly on absorption perturbations, since they are directly correlated to concentrations changes of oxy- and deoxyhemoglobin which are relevant both in functional imaging and in pathological conditions like in breast [

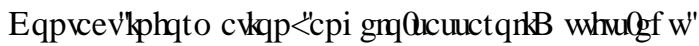

Optical Tomography and Spectroscopy of Tissue IX,

Edited by Bruce J. Tromberg, Arjun G. Yodh, Mamoru Tamura, Eva M. Sevick-Muraca, Robert R. Alfano, Proc. of SPIE Vol. 7896, 78961N · C 2011 SPIE · CCC code: 1605-7422/11/\$18 · doi: 10.1117/12.876943 
cancer. Perturbation theory of absorption defects lends itself to be reframed by using the method of the pathlength moments. In this way, the developed theory has more physical or statistical connotations than other approaches to perturbation theory. It is exactly this physical "flavor" of our approach to perturbation theory, which allowed us to develop approximated formulas that are grounded on a rigorous theory and have also an intuitive physical meaning. Two are the important results of our theory: a) we show that it is possible to develop a fast and efficient forward problem solver in frequency and time domains (the latter is usually computationally challenging), at least in those geometries where the Green's function of the medium is available; b) we show that the most important physical parameters needed for higher order perturbation theory are the time-resolved partial mean pathlengths $\left\langle l_{i}\right\rangle(t)$ in the regions of interest ( $i$ $=1,2, . . N)$. We claim that in several situations, the problem of perturbation theory in an arbitrary geometry is solved once the moments $<l_{i}>(t)$ are estimated.

\section{THEORY}

In a scattering medium where the microscopic Beer-Lambert law is valid, we can write the solution of a general forward problem for absorbing perturbations by using the formula:

$$
\begin{aligned}
& R_{f i}\left(\mathbf{r}_{b}, t, \boldsymbol{\mu}_{\boldsymbol{a}}+\Delta \boldsymbol{\mu}_{\boldsymbol{a}}\right)=R_{i n}\left(\mathbf{r}_{b}, t, \boldsymbol{\mu}_{\boldsymbol{a}}\right) \times \\
& \sum_{k_{1}, k_{2} \ldots k_{N}=0}^{\infty}(-1)^{k_{1}+k_{2}+\ldots k_{N}} \frac{\left(\Delta \mu_{a 1}\right)^{k_{1}} \ldots .\left(\Delta \mu_{a N}\right)^{k_{N}}}{k_{1} ! \ldots . k_{N} !}<l_{1}^{k_{1}} \ldots l_{N}^{k_{N}}>\left(\mathbf{r}_{b}, t, \boldsymbol{\mu}_{\boldsymbol{a}}\right)
\end{aligned}
$$

where $R_{i n}\left(\mathbf{r}_{b}, t, \boldsymbol{\mu}_{a}\right)$ and $R_{f i}\left(\mathbf{r}_{b}, t, \boldsymbol{\mu}_{a}+\Delta \boldsymbol{\mu}_{a}\right)$ are the temporal point spread functions (TPSF) calculated at the detector's location $\left(\mathbf{r}_{b}\right)$ in the initial and final states of the medium (characterized by a different distribution of the absorption coefficients), respectively, and $<l_{1}^{k_{1}} \ldots l_{N}^{k_{N}}>\left(\mathbf{r}_{b}, t, \boldsymbol{\mu}_{a}\right)$ is the time-domain mixed pathlength moment of order $\left(k_{1}, k_{2}, \ldots . ., k_{N}\right)$ calculated in the initial state of the medium and defined by the formula:

$$
<l_{1}^{k_{1}} \ldots l_{N}^{k_{N}}>\left(\mathbf{r}_{b}, t, \boldsymbol{\mu}_{a}\right)=\frac{\int_{\mathbf{\sigma}^{N}}\left(v t_{1}\right)^{k_{1}} \ldots\left(v t_{N}\right)^{k_{N}} f_{\text {in }}\left(t_{1}, \ldots . t_{N}\right) d \boldsymbol{\sigma}}{\int_{\boldsymbol{\sigma}^{N}} f_{i n}\left(t_{1}, \ldots . t_{N}\right) d \boldsymbol{\sigma}} .
$$

In Eqs. (1) and (2), $l_{i}=v t_{i}$ is a random variable associated with the pathlength traveled by a detected photons in the region " $i$ " and $\mathbf{r}_{b}$ is the detector's location. In Eq. (2) $f_{i n}\left(t_{1}, \ldots t_{N}\right)$ is the generalized temporal point spread function which is defined as the probability density for a detected photon to spend the time " $t i_{i}$ " in the region " $i$ "; while the region of integration $\left(\sigma^{N}\right)$ is the hyperplane in $R^{N}$ defined by: $\sum_{i=1}^{N} t_{i}=t$. In Fig. 1 we show a schematic diagram of the scattering medium having homogeneous initial values of the optical properties $\left(\mu_{a b}, \mu_{s b}^{\prime}\right)$ and $N=4$ regions of interest where absorbing perturbations are localized. Note that the theory is valid for a general distribution of the reduced scattering coefficient, with the only assumption that it does not change between the initial and final states of the medium. In our previous work, ${ }^{11}$ we have found a way to calculate the moments by using a formal similarity between Eq. (1) and the solution of the Fredholm equation of the II kind which is derived from the perturbation theory of the DE. For example, the $n^{\text {th }}$ order self moment in continuous-wave (CW) domain was found as:

$$
<l_{i}^{n}>\left(\mathbf{r}_{b}, \boldsymbol{\mu}_{a}\right) \approx \frac{n !<l_{i}>\left(\mathbf{r}_{b}, \boldsymbol{\mu}_{a}\right)}{V_{i}} \int_{V_{i}} d \mathbf{r}_{1} \int_{V_{i}} \phi_{0}\left(\mathbf{r}_{1}, \mathbf{r}_{2}\right) d \mathrm{r}_{2} \int_{V_{i}} \phi_{0}\left(\mathbf{r}_{2}, \mathbf{r}_{3}\right) \ldots \ldots d \mathbf{r}_{3} \int_{V_{i}} \phi_{0}\left(\mathbf{r}_{n-1}, \mathbf{r}_{n}\right) d \mathbf{r}_{n}
$$


where $<l_{i}>$ is the CW mean pathlength in the region " $i$ ", $V_{i}$ its volume and $\phi_{0}\left(\mathbf{r}_{i}, \mathbf{r}_{j}\right)$ is the Green's function for the fluence rate in the initial state of the medium calculated at $\mathbf{r}_{i}$ when the isotropic source is at $\mathbf{r}_{j}$.

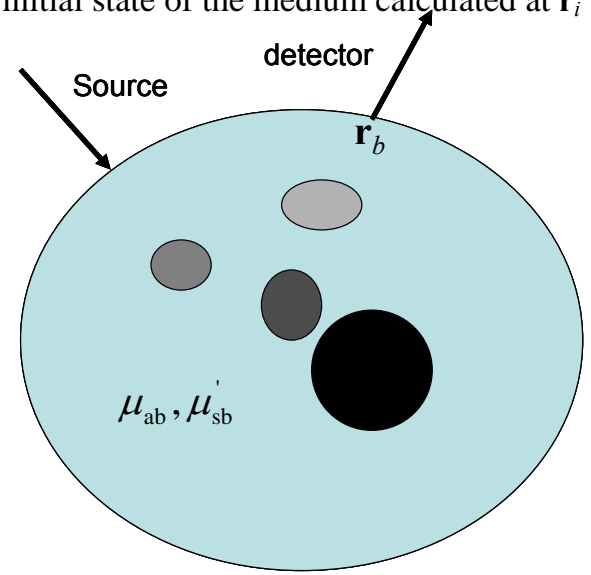

Fig. 1 Schematic representation of a scattering medium which includes four absorbing defects.

A dimensional analysis of the second term of Eq. (3) allowed us to derive the following heuristic formula:

$<l_{i}^{n}>\left(\mathbf{r}_{b}, \boldsymbol{\mu}_{a}\right) \approx c_{n-1}<l_{i}>\left(\mathbf{r}_{b}, \boldsymbol{\mu}_{a}\right)\left(\int_{V_{i}} \phi_{0}\left(\mathbf{r}, \mathbf{r}_{i}\right) d \mathbf{r}\right)^{n-1}, n>1$

where $\phi_{0}\left(\mathbf{r}, \mathbf{r}_{i}\right)$ is the Green's function of the fluence rate corresponding to the optical properties of the background medium and relative to a fixed source point inside the defect $\left(\mathbf{r}_{i}\right)$. We note that in all above formulas, the subscripts " 0 " and "in" have the same meaning and are used interchangeably. The dimensionless parameters $c_{n-1}$ were calculated when the source point was located at the center of the defect. Surprisingly, these parameters have shown very little variability, regardless of the geometry of the medium, optical properties, size and location of the defect. ${ }^{12}$ Therefore they can be considered as quasi-constant scaling parameters (characteristic of photon migration in diffusing media) which can be used for deriving more precise values of the higher order moments once the first order moments are known. In all the studies, we have used: $c_{1}=1.53, c_{2}=3.4$ and $c_{3}=10$. In other works, ${ }^{14,15}$ we have noticed that the structure of Eq. (4) shows the dependence of the higher order moments upon two factors: the first one $\left(<l_{i}>\right)$ which depends on more global optical properties, and the second factor (the integral) which depends on more local optical properties at the defect location. This observation allowed us to find simple solutions for the $\mathrm{DE}$ also for initial media that were not homogeneous. To our knowledge, these were the first results showing the application of perturbation theory to non-homogeneous background media.

In a more recent work, ${ }^{13}$ we have expanded the results of the proposed perturbation theory also to the frequency and time domains. The formulas which correspond to Eq. (4) are:

$$
\begin{array}{ll}
<l_{i}^{n}>\left(\mathbf{r}_{b}, t\right) \approx \frac{c_{n-1}}{R_{i n}\left(\mathbf{r}_{b}, t\right)}\left\{\left[R_{i n}\left(\mathbf{r}_{b}, \tau\right)<l_{i}>\left(\mathbf{r}_{b}, \tau\right)\right] \bigotimes_{j=1}^{n-1} \int_{V_{i}} \phi_{0}\left(\mathbf{r}, \mathbf{r}_{i}, \tau\right) \mathrm{d} \mathbf{r}\right\}(t) & 2 \leq n \\
<l_{i}^{n}>\left(\mathbf{r}_{b}, \omega\right)=c_{n-1}<l_{i}>\left(\mathbf{r}_{b}, \omega\right)\left(\int_{V_{i}} \widetilde{\phi}_{0}\left(\mathbf{r}, \mathbf{r}_{i}, \omega\right) \mathrm{d} \mathbf{r}\right)^{n-1} \quad 2 \leq n
\end{array}
$$


in the time domain and frequency domain, respectively. We note that in Eqs. (5) and (6) the dependence of the higher order moments upon the initial distribution of the absorption coefficient has been omitted. In Eq. (5) the function inside square brackets is convolved " $n$ - 1 " times with the same temporal function (the integral). Also, the integrands in both formulas are the Green's function in the time domain (Eq. (5)) and frequency domain (Eq. (6)). The time-domain mean pathlength, $\left\langle l_{i}>\left(\mathbf{r}_{b}, t\right)\right.$, has a direct physical meaning: it is the average pathlength traveled by a photon inside the region " $i$ " and detected at the boundary point $\mathbf{r}_{\mathrm{b}}$, at time $t$. Therefore, the time domain mean pathlength is a function of the arrival time and in general depends on the distribution of the optical properties of the medium and also on the source-detector separation. However, two important properties are derived in two particular cases: a) for media having a homogeneous absorption coefficient, $\left\langle l_{i}>\left(\mathbf{r}_{b}, t\right)\right.$ is independent on the value of the absorption coefficient; b) for media having a layered structure in the absorption coefficient and homogeneous reduced scattering coefficient, $<l_{i}>\left(\mathbf{r}_{b}, t\right)$ is independent on the source-detector separation. The first property is immediately derived from Eq. (2) by using the scaling property of the microscopic Beer-Lambert law with respect to the absorption coefficient; ${ }^{11}$ the second properties derives from the structure of the analytical solution of DE in layered media found with the eigenfunction method. ${ }^{16}$

The frequency-domain mean pathlength in Eq. (6) is defined by the formula: ${ }^{13}$

$$
<l_{i}>\left(\mathbf{r}_{b}, \omega\right)=\frac{F T\left[R_{i n}\left(\mathbf{r}_{b}, t\right)<l_{i}>\left(\mathbf{r}_{b}, t\right)\right](\omega)}{\widetilde{R}_{i n}\left(\mathbf{r}_{b}, \omega\right)}
$$

where the symbol " $F T$ " means the Fourier Transform and $\widetilde{R}_{i n}\left(\mathbf{r}_{b}, \omega\right)$ is the Fourier Transform of $R_{i n}\left(\mathbf{r}_{\mathrm{b}}, t\right)$. We note that the CW mean pathlengths are simply derived from Eq. (7) for $\omega=0$. As we can see from Eq. (7), the frequency-domain mean pathlengths are calculated once the time-domain mean pathlengths and Green's function relative to the background medium are known. The time-domain mean pathlenghts are calculated by the formula: ${ }^{13}$

$$
<l_{i}>\left(\mathbf{r}_{b}, t\right) \approx \frac{V_{i}}{R_{i n}\left(\mathbf{r}_{b}, t\right)}\left[R_{i n}\left(\mathbf{r}_{b}, \mathbf{r}_{i}, \tau\right) \otimes \phi_{0}\left(\mathbf{r}_{i}, \tau\right)\right](t)
$$

In the frequency domain, a formula formally identical to Eq. (1) holds, with the only substitution of the Green's function and the mixed moments in time domain with those in frequency domain. In our recent work, ${ }^{13}$ we have also derived useful expressions for the mixed moments $\left(<l_{i}^{k_{i}} l_{j}^{k_{j}} . . l_{N}^{k_{N}}>\left(\mathbf{r}_{b}, t\right)\right.$, for $\left.\sum_{i} k_{i} \leq 4\right)$. The frequency-domain mixed moments are defined from the timedomain mixed moments by using Eq. (7) with the substitution of $<l_{i}^{k_{i}} l_{j}^{k_{j}} . . l_{N}^{k_{N}}>\left(\mathbf{r}_{b}, t\right)$ instead of $<l_{i}>\left(\mathbf{r}_{b}, t\right)$. Finally the changes in $A C$ and phase ( $\alpha$ ) corresponding to a change in the absorption coefficient are defined by the formulas: ${ }^{13}$

$$
\begin{aligned}
& \frac{\Delta A C\left(\mathbf{r}_{b}, \omega, \boldsymbol{\mu}_{a}\right)}{A C_{0}\left(\mathbf{r}_{b}, \omega, \boldsymbol{\mu}_{a}\right)}=\frac{\left|\tilde{R}_{f i}\left(\mathbf{r}_{b}, \omega, \boldsymbol{\mu}_{a}+\Delta \boldsymbol{\mu}_{a}\right)\right|-\left|\widetilde{R}_{i n}\left(\mathbf{r}_{b}, \omega, \boldsymbol{\mu}_{a}\right)\right|}{\left|\tilde{R}_{i n}\left(\mathbf{r}_{b}, \omega, \boldsymbol{\mu}_{a}\right)\right|} \\
& \Delta \alpha\left(\mathbf{r}_{b}, \omega, \boldsymbol{\mu}_{a}\right)=\arg \left[\tilde{R}_{f i}\left(\mathbf{r}_{b}, \omega, \boldsymbol{\mu}_{a}+\Delta \boldsymbol{\mu}_{a}\right)\right]-\arg \left[\tilde{R}_{i n}\left(\mathbf{r}_{b}, \omega, \boldsymbol{\mu}_{a}\right)\right]
\end{aligned}
$$

As for the methods of Padé Approximants and Monte Carlo (MC), we refer to our recent publications. ${ }^{13,14}$ We only remind that the method of Padé approximants is not independent from the fourth order 
perturbation theory, since it relies on the correct computation of the moments. In other words, the prerequisite for the Padé approximant method to work is that we calculate correctly the values of the moments. We remind also that MC data are considered to be "gold standard" in the following simulation results.

\section{RESULTS}

We illustrate the performance of the developed perturbation theory and Padé Approximants method with a typical example in each domain of investigation.

\section{a) Time domain}

In Fig. 3 are shown some results relative to the slab geometry of Fig. 2 . The changes of the TPSF $\left(\Delta R / R_{0}=\right.$ $\left(R_{f i}-R_{\text {in }}\right) / R_{i n}$; see Eq. (1)) are plotted against the arrival time of detected photons for two values of the absorption contrast of the spherical inclusions (which is the same for both inclusions): $\Delta \mu_{a}=0.025 \mathrm{~mm}^{-1}$ (panels (a) and (b)), $\Delta \mu_{a}=0.055 \mathrm{~mm}^{-1}$ (panel (c)).

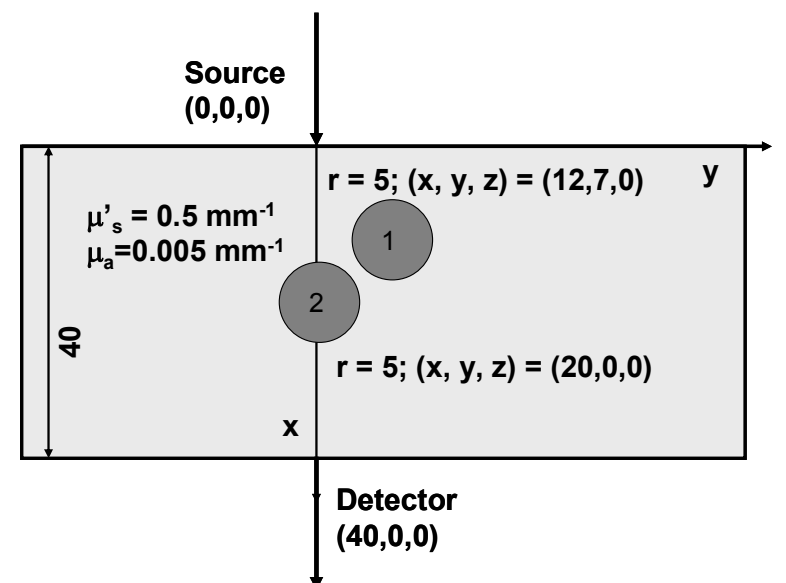

Fig.2 Schematics of the slab geometry including two absorbing defects. Geometrical units are in mm.


Fig. 3 The changes of TPSF with respect to that of the background medium are plotted against the arrival time of detected photons for two different values of the absorption contrast of the defects in Fig. $2: \Delta \mu_{a}=0.025 \mathrm{~mm}^{-1}$ (panels 
(a) and (b)), $\Delta \mu_{a}=0.055 \mathrm{~mm}^{-1}$ (panel (c)). The plots refer to four different orders of perturbation theory, to MC results (thick curves) and to the Padé method.

As we can see from Fig. 3, the performance of perturbation theory increases with the order of approximation considered. We note that for "smaller" values of the absorption contrast (Fig. 3(a)) even a fourth order approximation yields good comparison with MC data. However, for higher values of the absorption contrast, only the method of Padé approximants shows excellent comparison with MC data (Fig. 3(c)).

\section{b) Frequency domain}

The changes in $A C$ intensity and phase relative to the background values are plotted against the absorption contrast of the spherical defects in Fig. 2.
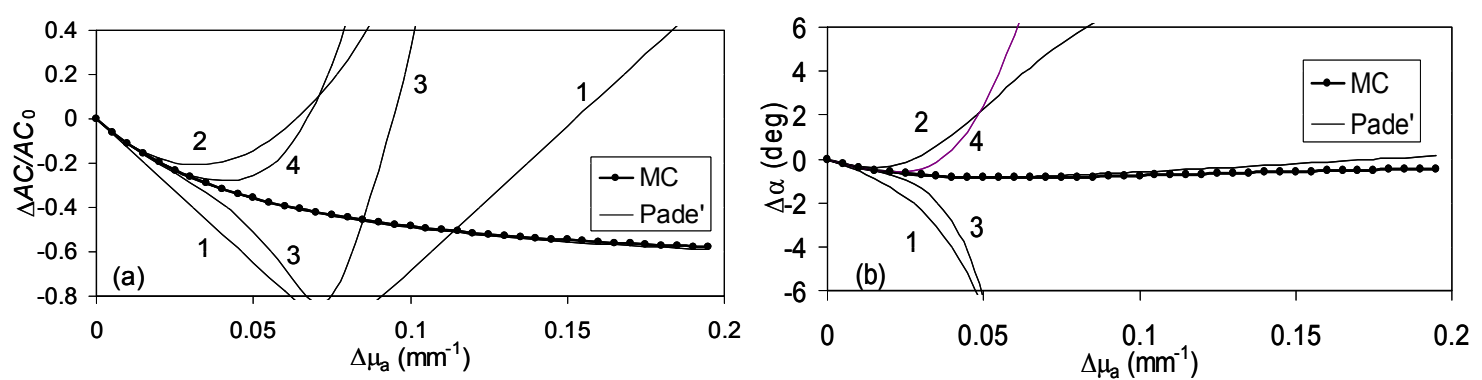

Fig. 4 Changes of $A C$ (panel (a)) and phase (panel (b)) data with respect to the background values for the situation of Fig. 2. The curves refer to the four orders of approximations, Padé approximants and MC data.

Also in the frequency domain, as it is expected, the accuracy of perturbation theory increases with the order of the approximation. The method of Padé approximants shows the best match with MC data for a wide range of absorption contrasts. These comparisons and others (not shown in this work) prove the excellent performance of our proposed perturbation theory also in the frequency domain.

\section{CONCLUSIONS}

In this work, we have shown that it is possible to develop practical solutions of fourth order perturbation theory for absorbing defects also for time domain and frequency domain. The method developed is based on approximate formulas for the calculation of higher order pathlength moments. The fourth order approximation yields correct calculation of the output flux for a wide range of absorption contrast, which cover not only typical values of interest in functional brain studies, but also in pathological conditions like breast cancer. If we want to estimate the perturbation in the output flux due to higher absorbing structure, e.g. a blood vessel, the method of Padé approximants must be used. We have recently applied this method for the modeling of the perturbation induced by vein motion and dilation. ${ }^{17}$ The proposed heuristic formulas for the calculation of higher order moments lighten up the computational burden of higher order perturbation theory. For example, all the calculations in the time domain were carried out with a computational time that did not exceed about 15 seconds on a Pentium IV 1.66 GHZ personal computer. This CPU time was necessary to calculate 5000 points of a TPSF. We have shown that higher order self moments in each domain of investigation, can be easily derived once the time domain mean pathlengths are known. Another important point is that, in some situations, the mixed moments (which are much more numerous than the self moments, given a certain number $N$ of regions of interest) give a negligible contribution to the calculation of the perturbed output flux. ${ }^{13}$ This finding is worth further investigation because it may open up the possibility to use our developed perturbation theory to more general situations. In the future, we are planning to apply the proposed method to in vivo data for the measurement of 
localized hemoglobin concentrations associated with pathological conditions and physiological or functional processes.

\section{Acknowledgments:}

This research is supported by NIH Grant R01-NS059933. F. Martelli acknowledges funding from the European Community's Seventh Framework Program [FP7/2007-2013] under grant agreement $n^{\circ}$. HEALTH-F5-2008-201076.

\section{REFERENCES}

[1] Kienle, A., Glanzmann, T., Wagnieres, G., and Bergh, H. V., "Investigation of two-layered turbid medium with time-resolved reflectance,” Appl. Opt. 37, 6852-6862 (1998).

[2] Martelli, F., Sassaroli, A., Del Bianco, S., Yamada, Y., Zaccanti, G., "Solution of the timedependent diffusion equation for layered diffusive media by the eigenfunction method," Phys. Rev. E 67056623 1-14 (2003).

[3] Arridge, S. R., Schweiger, M., Hiraoka, M., Delpy, D.T., “A Finite Element approach to modelling photon transport in tissue,” Med. Phys. 20, 299-309 (1993).

[4] Dehghani, H., Eames, M. E., Yalavarthy, P. K., Davis, S. C., Srinivasan, S., Carpenter, C. M., Pogue, B. W., Paulsen, K. D., "Near infrared optical tomography using NIRFAST: Algorithm for numerical model and image reconstruction,” Commun. Numer. Meth. Engng. 25 711-732 (2009).

[5] Bluestone, A. Y., Abdoulaev, G., Schmitz, C. H., Barbour, R. L., Hielscher, A. H., "Threedimensional optical tomography of hemodynamics in the human head," Optics Express, 9 272-286 (2001).

[6] Ren, K., Abdoulaev, G., Bal, G., Hielscher, A. H., "Frequency-domain optical tomography based on the equation of radiative transfer," SIAM Journal on Scientific Computing 28 1463-1489 (2006).

[7] Ostermeyer, M. R., and Jacques, S. L., "Perturbation theory for diffuse light transport in complex biological tissues,” J. Opt. Soc. Am. A 14, 255-261 (1997).

[8] Boas, D. A., "A fundamental limitation of linearized algorithms for diffuse optical tomography," Opt. Exp. 1, 404-413 (1997).

[9] Grosenick, D., Kummrow, A., Macdonald, R., Schlag, P. M., Rinneberg, H., "Evaluation of higherorder time domain perturbation theory of photon diffusion on breast-equivalent phantoms and optical mammograms,” Phys. Rev. E 76, 061908 1-18 (2007).

[10] Wassermann, B., "Limits of high-order perturbation theory in time-domain optical mammography,” Phys. Rev. E. 74, 031908 1-13 (2006)

[11] Sassaroli, A., Martelli, F., Fantini, S., "Perturbation theory for the diffusion equation by use of the moments of the generalized temporal point-spread function: I. Theory,” J. Opt. Soc. Am. A 23, 2105-2118 (2006).

[12] Sassaroli, A., Martelli, F., Fantini, S., "Perturbation theory for the diffusion equation by use of the moments of the generalized temporal point-spread function: II. Continuous wave results,” J. Opt. Soc. Am. A 23, 2119-2131 (2006).

[13] ] Sassaroli, A., Martelli, F., Fantini, S., "Perturbation theory for the diffusion equation by use of the moments of the generalized temporal point-spread function: III. Frequency-domain and timedomain results,” J. Opt. Soc. Am. A 27, 1723-1742 (2010).

[14] Sassaroli, A., Martelli, F., Fantini, S., "Fourth order perturbation theory for the diffusion equation: continuous wave results for absorbing defects," SPIE Proceedings 7174, 717402 1-12 (2009).

[15] Sassaroli, A., Martelli, F., Fantini, S., "Higher order perturbation theory for the diffusion equation in heterogeneous media: application to layered and slab geometries,” Appl. Opt. 48, D62-D73 (2009).

[16] Martelli, F., Sassaroli, A., Yamada Y., Zaccanti G., "Analytical approximate solutions of the timedomain diffusion equation in layered slabs,” J. Opt. Soc. Am. A 19, 71-80 (2002).

[17] Chen, D. K., Erb, M. K., Tong, Y., Yu, Y., Sassaroli, A., Bergethon, P. R., Fantini, S., "Spectral and Spatial Features of Diffuse Optical Signals in Response to Peripheral Nerve Stimulation,"

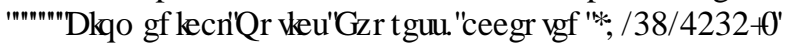

